\title{
Influence of background carriers on magnetic properties of Mn-doped dilute magnetic $\mathrm{Si}$
}

\author{
S. Mukhopadhyay ${ }^{a, c}$ and Nicholas M. Harrison ${ }^{b, c}$ \\ ${ }^{a}$ Department of Materials, ${ }^{b}$ Department of Chemistry, ${ }^{c}$ Thomas Young Center: The \\ London Center for Theory and Simulation of Materials, Imperial College London, \\ Exhibition Road, London, SW7 2AZ, United Kingdom
}

\begin{abstract}
Using hybrid exchange density functional calculations we show that the type of background carriers has profound effects on magnetic interactions in Mn doped dilute magnetic Si. The p- and n-type Si were simulated by introducing an extra hole and an extra electron, respectively in the 64 atoms $\mathrm{Si}$ supercell. In case of p-type Si compensated by a homogeneous background potential and $1.6 \% \mathrm{Mn}$, the ground state is ferromagnetic, whereas other conditions remaining the same, the ground state becomes antiferromagnetic for the n-type Si. The exchange energies in Mn-doped extrinsic Si are higher by about $1 \mathrm{eV} / \mathrm{Mn}$ atom compared to the Mn doped intrinsic Si. Calculated electronic structures reveal that in p-type $\mathrm{Si}: \mathrm{Mn}$ the hole localises over $\mathrm{Mn}$ and the short range magnetic coupling increases. Our calculations indicate that localisation of magnetic polarons at the Mn site is likely, which, in turn enhances long range magnetic interaction between Mn ions and responsible for FM stabilisation. On the other hand, in the n-type host electron-electron repulsion increases within Mn-Si impurity band and the short range coupling decreases, which destroys the long range spin polarisation. These calculations explain the observed ferromagnetism in the p-type Si:Mn at higher temperatures than in the n-type Si:Mn and the magnetic moments of the systems compare well with experiments.
\end{abstract}

Keywords: Spintronics, Dilute magnetic Silicon, Magnetism, Silicon, Mn doped Silicon

PACS: 85.35.-p, 73.40.Gk, 73.61.Ng, 3.40.Qv, 73.40.Rw 


\title{
Influence of background carriers on magnetic properties of Mn-doped dilute magnetic Si
}

\author{
S. Mukhopadhyay ${ }^{a, c}$ and Nicholas M. Harrison ${ }^{b, c}$ \\ ${ }^{a}$ Department of Materials, ${ }^{b}$ Department of Chemistry, ${ }^{c}$ Thomas Young Center: The \\ London Center for Theory and Simulation of Materials, Imperial College London, \\ Exhibition Road, London, SW7 2AZ, United Kingdom
}

\section{Introduction}

Dilute magnetic semiconductors (DMS) have the potential for spintronic applications because manipulations of spins (1) are possible in these materials. The mature state of silicon technology offers high incentives for making silicon based DMS. This makes magnetically active transition metal impurities in Si important. Mn is particularly interesting as a magnetic dopant because of the report of room temperature ferromagnetism in Si:Mn (2). Although transition metal doped III-V semiconductors have been investigated extensively $(3 ; 4 ; 5 ; 6 ; 7 ; 8 ; 9)$, the nature of magnetic interaction in group IV materials is a matter of controversy. The first experimental observation in Mn implanted dilute magnetic Si reported room temperature ferromagnetism (2), however, subsequent reports show wide range of Curie temperatures (Tc) , from $70 \mathrm{~K}$ to $400 \mathrm{~K}$, depending on the preparation conditions, annealing temperatures and in particular the type of background carrier (10; 11; 12; 13; 14).

For the design of DMS, understanding the mechanism of low Tc is very important. Tc is much lower than room temperature due to difficulty in achieving percolation of the ferromagnetic coupling, which, in turn, is caused by sort range magnetic interaction (15). Due to the low solubility of transition metals in the DMSs, they show spinosoidal decomposition or phase separation at thermal equilibrium. This causes high blocking temperature, which is important for high Tc DMSs. It has been shown by the Monte Carlo method for the Ising model that spinodal nano-decomposition with high blocking temperatures is important for a state of "Super-paramagnetism" in DMSs. (16; 17; 18; 19; 20). 
In the first report of ferromagnetic Si:Mn (2) it has been shown that room temperature ferromagnetism is the property of p-type Si but not of n-type one., however, the role of positive charge carrier is not well understood. This indicates the urgent requirement of a detailed investigation on the electronic and magnetic interaction and the role of the background carrier type in $\mathrm{Mn}$ doped Si.

Previous theoretical investigations have considered Mn impurity in intrinsic Si $(21 ; 22,23,24 ; 25 ; 26 ; 27)$. Two very recent studies have revealed (28; 29) by first principle calculations using plane wave pseudopotential that the site preference of the Mn dopant is changed dramatically from interstitial to substitutional if an n-type impurity is present nearby. The result establishes the very strong influence of the type background carrier as both the electronic structure and the physical structure of the Mn doped Si. In this calculation they have used the dopant atoms to create background charge, therefore, it is not clear whether the background carrier or the ionic interaction between Mn and the dopant is responsible for this result. The observed increase in Tc upon p-doping could be due to the presence of holes in the top of the valence band or due to the stabilisation $\mathrm{Mn}$ in its interstitial position.

In the current investigation we have addressed the problem of background carrier by considering uniform background charge carrier, $\mathrm{p}$ or $\mathrm{n}$ type, in order to separate out the effect of ionic interaction in between the background dopant and Mn. To isolate the position aspect on electronic structure we consider the Mn substituted Si only. This work has brought out the essential relationship between magnetic coupling in presence of $\mathrm{p}$ and $\mathrm{n}$ type background carriers in Mn substituted Si.

\section{Methodology}

We performed electronic structure calculations using density functional theory as implemented in the CRYSTAL code (30). For computational simplicity one substitutional $\mathrm{Mn}$ atom has been considered in 64 atom Si supercell corresponding to Mn concentrations of 1.6\%. This also implies that the calculations presented in this paper do not assume any phase separation. The Mn concentration of $1.6 \%$ is the typical Mn concentration for Mn doped Si DMS (10; 11; 12; 14). however, most of the theoretical studies have used small supercell sizes or higher Mn concentrations (21; 22, 25; 26; 27). The CRYSTAL implements atom centered Gaussian basis set to construct Bloch functions. Triple valence with d polarisation functions Gaussian basis sets 
have been used for Si and Mn. The hybrid exchange functional (B3LYP) has been used which compensates for self-interaction errors inherent in the LDA and GGA functionals. Self interaction corrections (SIC) have been previously shown to be essential for the correct description of DMS based on III-V and oxide systems $(7,8 ;$; 9$)$. This functional has been shown to give reliable energetics, electronic structures, photoemission spectra and magnetic properties in a variety of semiconductors and strongly correlated systems (8; 9; 31; 32).

The integrations in reciprocal space have been carried out on a PackMonkhurst grid corresponds to $35 \mathrm{k}$-points in the irreducible part of the Brillouin zone and 165 points in Gilate net. Full geometrical relaxations, for unit cell sizes and internal coordinates, have been performed using the BFGS method as implemented in the CRYSTAL code (30). Geometrical relaxations are considered complete when residual forces are below $5.5 \mathrm{meV} / \AA$. The effect of p- type (or n-type) doping has been simulated by employing one extra positive (or negative) charge in a 64 atom unit cell compensated by constant background potential to maintain charge neutrality. This gives a background carrier concentration of about $10^{20} \mathrm{~cm}^{-3}$, which is much higher than typical experimental concentrations of about $10^{15} \mathrm{~cm}^{-3}$ (12). Reduction of background carrier concentration requires larger super cell sizes, which has not been considered in current calculations. Calculations for antiferromagnetic (AFM) configurations were done by doubling the unit cell. Magnetic coupling energies $(\Delta \mathrm{E})$ are calculated by, $\Delta \mathrm{E}=\mathrm{E}_{A F M}-\mathrm{E}_{F M}$, where $\mathrm{E}_{A F M}$ and $\mathrm{E}_{F M}$ were the total energies for the AFM and the FM spin configurations per Mn atom, respectively.

\section{Results and Discussions}

The ground states obtained from our calculations for three different types of Si hosts, intrinsic, p-type and n-type, along with the magnetic coupling energies, magnetic moments on Mn atoms and on nearest neighbour Si atoms are listed in Table 1. For the intrinsic and the p-type Si:Mn the ground states are FM, but for the n-type host it is AFM. Magnitudes of magnetic coupling energies for the p-type host and for the n-type host are $1.27 \mathrm{eV}$ and $0.91 \mathrm{eV}$ ,respectively, higher than that in the intrinsic host.

Looking at magnitudes of magnetic moments on Mn ions from Table 1 it is found that for the n-type and the intrinsic hosts these are similar, but for the p-type host, the magnetic moment on Mn increases by $7.5 \%$ compared to that in the intrinsic host. It is useful to compare these results with reported 
Table 1: Magnetic ground states and magnetic moments for intrinsic, $\mathrm{p}$ and $\mathrm{n}$ type Si host with $1.6 \% \mathrm{Mn}$ concentrations .

\begin{tabular}{|c|c|c|cccc|}
\hline $\begin{array}{c}\text { Conduc- } \\
\text {-tivity } \\
\text { type }\end{array}$ & Ground & Mag. & \multicolumn{4}{|c|}{ Magnetic moments } \\
& & $\begin{array}{c}\text { coupling } \\
\text { energies } \Delta E\end{array}$ & $\mathrm{Mn}$ & $1 \mathrm{nn}$ & $2 \mathrm{nn}$ & Total/ \\
$\mathrm{eV}$ & $\mu_{B}$ & $\mu_{B}$ & $\mu_{B}$ & $\mu_{B}$ \\
\hline Intrinsic & FM & 0.58 & 4.12 & -0.19 & 0.001 & 3.0 \\
p-type & FM & 1.75 & 4.38 & -0.42 & -0.012 & 2.0 \\
n-type & AFM & -1.39 & 4.13 & -0.09 & 0.007 & - \\
\hline
\end{tabular}

theoretical results, however, no calculation of the magnetic moments on $\mathrm{Mn}$ ion in the $\mathrm{p}$ - and the n-type hosts are available for $1.6 \% \mathrm{Mn}$ concentration for comparison. For the intrinsic Si host, the calculated magnetic moment obtained by plane wave GGA method (23) is $52 \%$ lower than that obtained by the current calculations. The discrepancy may be due to the use of B3LYP functional in the current case.

Compared to the case of the intrinsic Si host, magnetic moments on first neighbour (1nn) Si atoms in the p-type host are more than doubled, and are halved in the n-type host. They are aligned antiferroganetically with $\mathrm{Mn}$ ions irrespective of the carrier type of the host. This result is consistent with other theoretical results obtained by FPLAPW-LSDA (27) and plane wave-GGA (26) methods, but the exact comparison is not possible because of their small cell size and higher concentrations of Mn (3.1\%).

Magnetic moments on second neighbour (2nn) Si atoms are two orders of magnitude smaller in the intrinsic and the n-type host and one order of magnitude smaller in the p-type host. In the p-type host the alignment of spin is FM with that of $1 \mathrm{nn} \mathrm{Si}$, however, for the intrinsic and n-type hosts those alignment is AFM. Magnetic moments on higher order Si neighbours are an order of magnitude smaller than that on $2 \mathrm{nn}$ Si.

The total magnetic moment per Mn ion per unit cell of the p-type host decreases by half of the magnetic moment on individual Mn ion because the increase of the spin-down magnetic moments over $1 \mathrm{nn}$ and $2 \mathrm{nn}$ Si ions. This total magnetic moment for the case of intrinsic Si host is compared well with the theoretical result obtained by plane wave GGA (28) calculations. For the p-type Si host this agrees with experimental magnetic moment ob- 
tained by SQUID magnetometer experiment (2), which has been obtained for about $0.8 \% \mathrm{Mn}$ concentration and $10^{19} \mathrm{~cm}^{-3}$ p-type background carrier concentration.

Therefore, from the above results it is found that ground state properties of the Si:Mn system strongly depend on the type of background carrier.

To understand the effect of background-carriers on the magnetic stabilisation we analyse electronic structures of these systems. Spin density diagrams obtained from our calculations for intrinsic, $\mathrm{p}$ type and n-type hosts are given in Fig. 1. From these diagrams it is observed that the spin polarisation of the $1 \mathrm{nn} \mathrm{Si}$ ions is only significant and this is directional. The spin polarisation reduces with the increasing distance of the Si ions from the $\mathrm{Mn}$ ion. The increase in spin polarisation of $1 \mathrm{nn}$ Si ions in the p-type host and the decrease of that in the n-type host can also be seen. The long range spin polarisation of spins up and down over Si ions is also seen in Fig 1(d), although, that spin polarisation decay quickly. The result for intrinsic Si is consistent with other theoretical findings (21, 22).

The total, orbital projected for $\mathrm{Mn}$ ions and atom projected for $1 \mathrm{nn} \mathrm{Si}$ ions densities of states (DOS) for ground states are shown in Fig. 2. A gap opens in the minority spin, irrespective of the host carrier type, where the top of the valence bands and the bottom of the conduction bands originate from Si-2p symmetry and hybridised pd orbitals of $\mathrm{Mn}$ and Si, respectively, implying that the band gaps are of charge transfer type similar to GaN:Mn system (7).

For the intrinsic host, as shown in Fig. 2(a), a distinct, localised, Mn-t2g derived impurity band (shown by I on the middle panel of the figure) is found at the Fermi energy of the majority spin DOS making the resulting system as half metallic, because the Mn-3d impurity band contributing to the majority spin remains half filled but a semiconducting gap exist in the minority spin DOS. From the middle and the lower panel of the figure we see that the origin of this impurity band is d-sp hybridisation between $3 \mathrm{~d}^{-} \mathrm{t}_{2 g}$ orbitals of $\mathrm{Mn}$, and the 3sp orbitals of its tetrahedrally positioned four Si neighbours. This impurity band is located at $546 \mathrm{meV}$ above of the top of the valence band, which is compared well with the energy position of the Mn impurity band in Si, 390meV above from the top of the valence band, obtained by combined DLTS-EPR experiments (33) . The large separation of this narrow and hybridized impurity band from the host valence band indicates a strong coupling between Mn-3d and Si-3sp orbitals. The Mn-3d -Si-3sp hybridised antibonding states for minority spin electrons are empty and at the bottom 


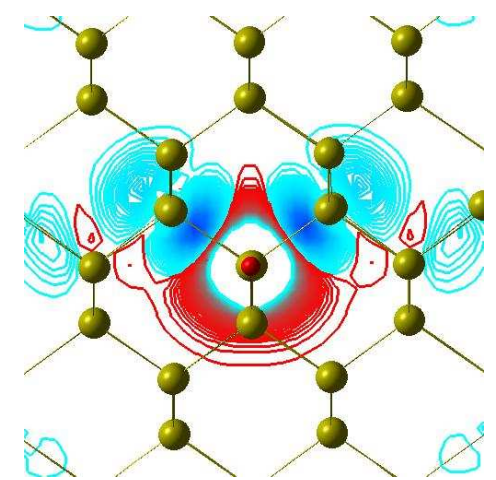

(a)

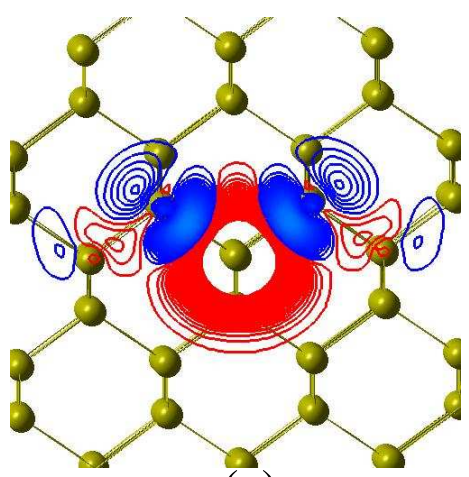

(c)

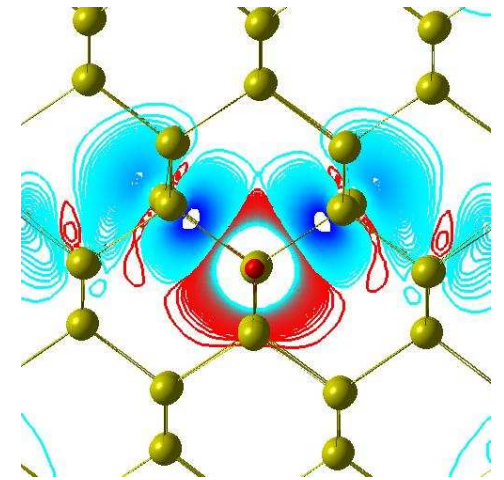

(b)

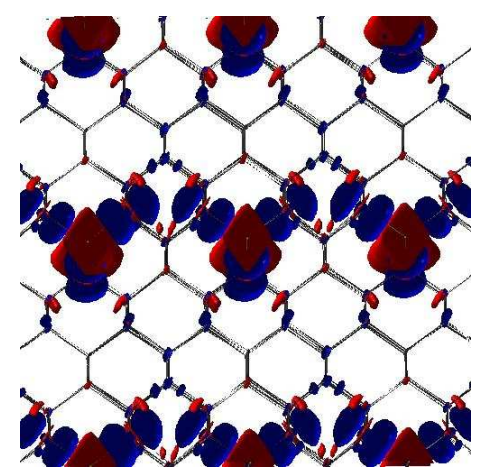

(d)

Figure 1: Spin densities on the 110 plane of the (a) intrinsic Si (b ) p-type Si, (c) n-type Si host for the range of $-0.02 \mu_{B} /$ bohr $r^{3}$ to $0.02 \mu_{B} /$ bohr $r^{3}$ with a step of $2 \times 10^{-4} \mu_{B} / b o h r^{3}$. The blue lines are for majority spin states and red lines are for minority spin states. The highest magnitude of spins for each type of spin is at the centre of contours. (d) The long range spin polarisation of the p-type Si:Mn. The range of this plot is $-0.02 \mu_{B} / b o h r^{3}$ to $+0.0002 \mu_{B} / b o h r^{3}$. The range has been modified from the former to include atoms having very small spins over them. 


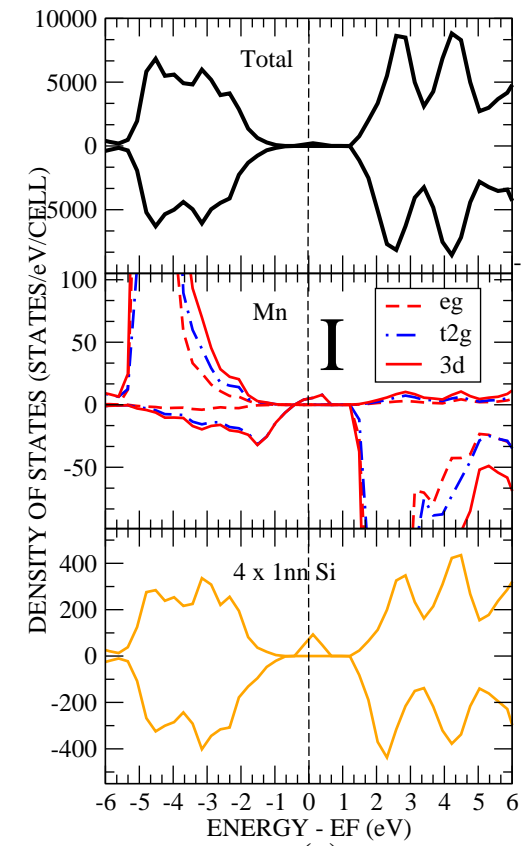

(a)

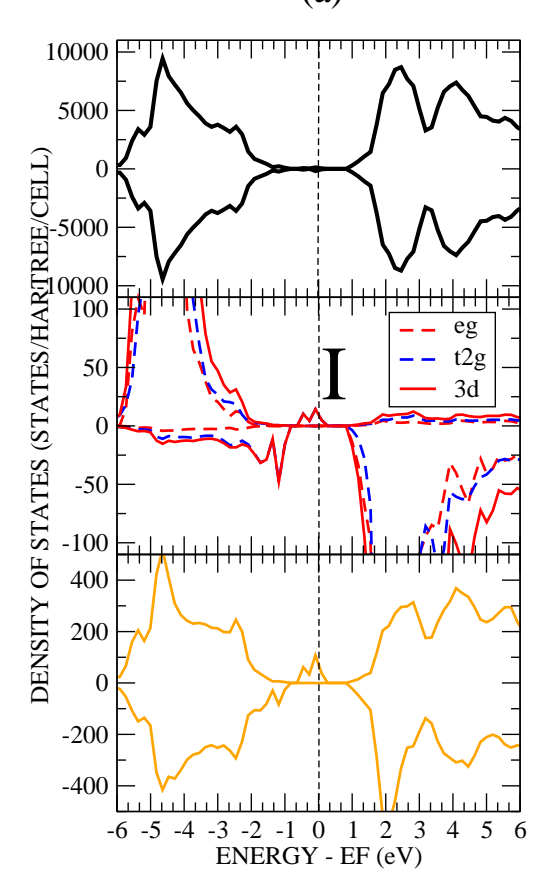

(c)

(c)

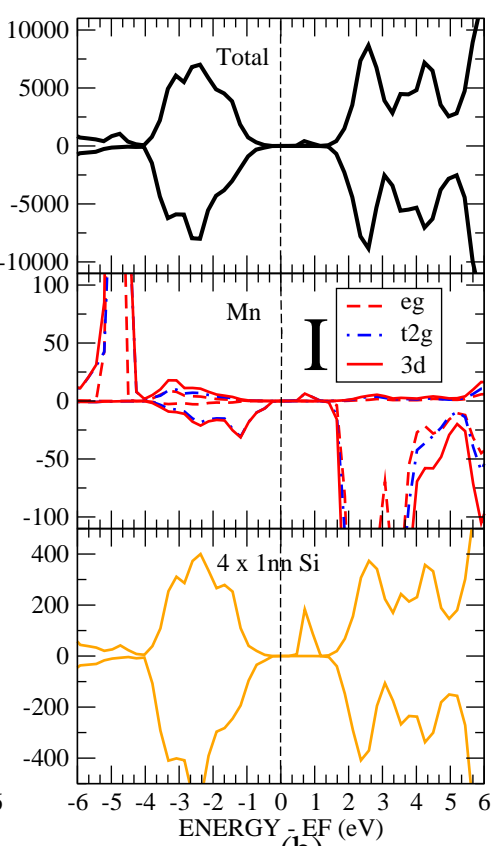

(b)
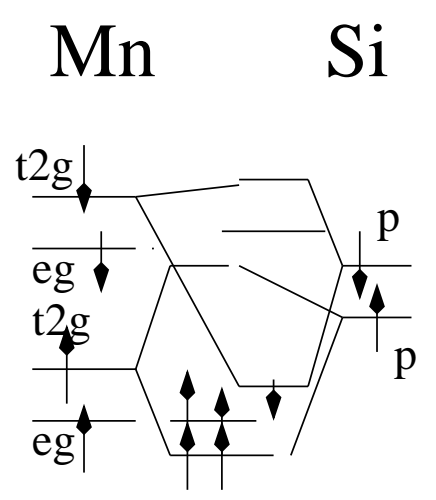

Figure 2: Densities of states (DOS) of (a) the intrinsic Si host, (b) the p-type Si host, (c) the n-type Si host. The black lines are for total DOS, red lines are for 3d orbital projected densities of states for Mn ions, and orange lines are for atom projected DOS for $1 \mathrm{nn}$ Si. The zero energy is the Fermi energy and positive (negative ) DOS are for majority (minority) spin. The position of the impurity level has been shown by I. For the n-type Si the orbital projected densities of states for one Mn ion has only been plotted. The simplified level filling scheme for the Mn doped in the p-type Si host is shown in (d). 
of the conduction band. This result is qualitatively consistent with other theoretical findings (21; 28; 23,22$)$, however, quantitative comparison is not possible because of different Mn concentrations (21; 22) or differences in exchange correlation potentials considered in those calculations.

Before discussing total DOS for the p-type and n-type Si host as shown in Fig. 2(b) and (c) it is worth to mention that in these calculations we have considered an extra electron or hole distributed evenly throughout the system to simulate the $\mathrm{n}$ or the $\mathrm{p}$ type host, respectively, therefore there is no extra ion present and no localised donor or acceptor energy level is expected. The localised Mn-t2g-Si-3sp derived impurity band (shown by I on the middle panel) must not be confused with states for the $\mathrm{p}$ or the $\mathrm{n}$ type background doping. Compare to the intrinsinc Si host (Fig 2(a)) Fermi energy for the p-type Si host (Fig 2(b)) decreases towards the valence band as expected for the p-type background carrier. In contrast to the intrinsic host, where the position of the the Fermi energy is at the middle of the impurity band, the Fermi energy for the p-type host is on the top of the valence band. This Si:Mn system opens a small band gap and is semiconducting. The d-sp hybridised isolated, localised and empty impurity band (I) situates $750 \mathrm{meV}$ above from the Fermi energy in the majority spin channel. From the middle and lower panel of the Fig 2 (b) we find that the overlap between 1nn Si-sp and Mn-3d reduces considerably from that in the intrinsic host. Compare to the intrinsinc Si host (Fig 2(a))this is the main difference that can be found. The Mn-3d peak of $t_{2 g}$ symmetry is $4 \mathrm{eV}$ below from the top of the valence band and Fermi energy, and is very narrow. This suggests that the Mn-Si bond in the p-type $\mathrm{Si}$ is more ionic than in the intrinsic host.

For the n-type Si:Mn, as shown in Fig. 2 (c), the Fermi energy is on the d-sp hybridised Mn-Si localised impurity band (I), similar to that found in the intrinsic Si host. There are two peaks have been found in this impurity band. The filled peak lowers its energy and localised more towards valence band and the partially empty peak is higher in energy by about $200 \mathrm{meV}$. This electronic structure is qualitatively similar to that found earlier for the n-type Si host (28) showing that the effect of co-doping with non magnetic impurity on the electronic structure is small.

The bond order analysis shows that Mn-Si bonds are covalent, which is similar to Si-Si bonds, for the intrinsic and the n-type host but the overlap is $16 \%$ smaller in the p-type host. The Mn-Si bond lengths are $2.482 \AA, 2.810 \AA$, and 2.426̊̊for the intrinsic, p-type and n-type Si host, respectively, showing that Mn-Si bond in the p-type host increases considerably. 
For the intrinsic Si host 0.12|e| charges transfer from Mn ions to nearest neighbour Si ions indicating that Mn ions are donors. This finding is consistent with the experimental observation by a combined DLTS-EPR experiment (33) that the Mn is a donor in Si host, when in substitutional site, but contrary to the claim of some others (28, 22), who considered Mn as an acceptor in the substitutional Si site. In the later case, however, authors didn't analyse any charge distribution.

For the n-type host the analysis shows that the introduced extra electron is not on the Mn ion but distributed among all Si ions. Nearest neighbour $\mathrm{Si}$ ions to the $\mathrm{Mn}$ ion receive $10 \%$ more electrons than those receive in an intrinsic Si host case, and from the second neighbour Si ions the electron occupation increases, but the occupation is not homogeneous.

For the p-type S:Mn case about $20 \%$ charge of the extra hole localised over Mn-3d orbital, but nearest neighbour Si ions are $30 \%$ more negative than they are in the intrinsic host. The remaining positive charge is distributed in-homogeneously over all other Si ions. The extra charge over 1nn Si ion changes its local geometry. Mn-Si bond length increases from its value in the intrinsic host as given before. The value of $\angle[\operatorname{Si}(2 n n)-\operatorname{Si}(1 n n)-\operatorname{Si}(2 n n)]$ increases by $4^{\circ}$ from its tetrahedral value $\left(109^{\circ}\right)$ and the value of the $\angle[\mathrm{Mn}-$ $\mathrm{Si}(1 \mathrm{nn})-\mathrm{Si}(2 \mathrm{nn})]$ decreases by the same. These angular distortions are $2^{\circ}$ in the intrinsic host from the pure $\mathrm{Si}$ and almost vanish in the n-type case.

From Mulliken population analysis for the intrinsic and n-type Si host show that there are 5.5 electrons in Mn-3d with 2 electrons in the $\mathrm{e}_{g}$ and 3.5 electrons in $\mathrm{t}_{2 g}$ implying that $\mathrm{Mn}$ ions are $\mathrm{Mn}^{2+}$. The extra 0.5 electron on $\mathrm{t}_{2 g}$ are due to back donation from nearest neighbour Si atoms due to covalent bonding. For the p-type host, Mulliken population analysis shows that there are 4.5 electrons in Mn-d with 2 electrons in the $\mathrm{e}_{g}$ and 2.5 electrons in the $\mathrm{t}_{2 g}$. This indicates that the charge state of $\mathrm{Mn}$ is $\mathrm{Mn}^{+3}$.

From the simplified level filling scheme as shown in Fig. 2(d) it is seen that the $t_{2 g}$ derived spin up level is less than half filled and the impurity band is empty. The spin-down antibonding level overlap with the $\mathrm{e}_{g}$ (down) level, which is not shown in the level diagram for clarity. The existence of spin-gap for both up and down spin is seen.

From these results it is found that for the intrinsic host the position of the Fermi energy is on the localised hybridised impurity band, consistent with the recent theoretical finding (28), and suggests that the indirect exchange may not be strong enough due to in-sufficient supply of conduction holes.

The situation is similar for the n-type host. The presence of the extra 
electron on Si ions reduces the transfer of spin polarised electrons from $\mathrm{Mn}$ ions to Si host, which leads to weak Mn-Si-Mn coupling, reduces the possibility of any indirect interaction and increases superexchange interaction. This type of interaction stabilises AFM configuration. The splitting of the impurity band for the n-type Si:Mn system, which is also found in other calculations (28) but not explained, shows that the strong electron-electron repulsion present within the impurity band. The magnitude of that Coulomb repulsion, which can be found from the energy difference of the splitted DOS peaks, compares well to the $200 \mathrm{meV}$ calculated by linear muffin tin orbital (LMTO) Greens function method. (33). The strong electron-electron repulsion within localised impurity band prevents the strong interaction with neighbouring $\mathrm{Si}$ and destroys long range spin polarisation as well as prevents the transfer of spin polarised electrons to nearest neighbour Si ions. This in turn also prevents FM ground state.

For the p type host, the localisaion of the extra hole on the Mn ion increases the Mn-3d-Si-3p coupling resulting the high exchange coupling energy given in Table 1, and increases transfer of spin-polarised electrons to nearest neighbour Si ions. The increase in coupling is also evident from the localised nature of the impurity band within the band gap, however, since this impurity band is empty and the overlap between Si-3sp and Mn-3d, as seen from Fig 2(b), is minimal, suggests that the coupling is ionic. Although the oscillatory nature of the Si spin orientation as shown in Fig 1 suggest the possibility of indirect exchange coupling, the insulating nature of the electronic structure indicates that no carrier will be available for the carrier mediated exchange interaction as claimed by other authors $(21 ; 26$; 27$)$. On the other hand the local geometrical distortion, localisation of the hole on the Mn ion, increase of its charge state, and the ionic nature of $\mathrm{Mn}$ and $\mathrm{Si}$ indicates that the magnetic polaron can be localised near the Mn ion. The localisation of the hole on Mn-3d makes one $\mathrm{Mn} \mathrm{t}_{2 g}$ electron available to transfer to $1 \mathrm{nn}$ $\mathrm{Si}$, which polarises not only $1 \mathrm{nn} \mathrm{Si}$ but also the $2 \mathrm{nn}$ as seen from Table 1 and the Fig 1(b). The long range spin polarisation as shown in Fig. 1(c) may be obtained due to the overlap of magnetic polarons centred on two Mn sites. This suggestion is consistent with the theoretical findings of possible magnetic polaron type interaction for Mn doped GaAs (34), although the concepts of magnetic polaron in Mn doped Si has not been reported yet. The large magnetic coupling energy in the p-type Si:Mn predicts the FM phase can be stable even at room temperature, which is consistent with experimental findings (2; 14). 
From these resultss we can speculate that that there may be an optimal concentration of the p-type background carrier, which can be suitable for magnetic Si. High concentrations of the p-type carrier will increase the opposite spin polarisations over $\mathrm{Si}$ ions, or in other words will increase the size of the magnetic polaron and will reduce the total magnetism. On the other hand, low concentrations of the p-type impurity will decrease ionic nature of Mn-Si magnetic coupling, delocalises magnetic polaron and therefore the exchange energy can be low enough to destroy the magnetic order. This explain why room temperature ferromagnetism has only been observed experimentally in the Mn doped p-type $\mathrm{Si}(2 ;$; 14$)$.

\section{Conclusions}

In summary our calculations predict that the magnetism in dilutely doped $\mathrm{Si}: \mathrm{Mn}$ is due to strong ionic magnetic-coupling between $\mathrm{Si}$ and Mn ions and governed by the magnetic polaron interaction in the long range leading to FM coupling of the Mn atoms. For the p-type background carrier, holes localise on Mn atoms increasing $\mathrm{p}-\mathrm{d}$ coupling in the short range and local geometrical distortion localises magnetic polaron. The long range magnetic interaction can be governed by overlapping magnetic polarons. The n-type background carrier, on the other hand, reduces the coupling and increases electron-electron repulsion within Mn-Si hybridised band. The latter is not suitable as magnetic Si. We speculate that a optimal p-type background carrier concentration can be achieved, which can facilitate magnetism. The calculated results agree well with experimental observations and provide useful information to designing magnetic semiconductors.

\section{Acknowledgements}

The computations have been done using the high performance computer system at Imperial College London, the SCARF computer system at STFC, Rutherford Appleton Laboratory and HPCx system, where computer resources were provided via our membership of the UK's HPC Materials Chemistry Consortium and funded by EPSRC (portfolio grant EP/D504872).

\section{References}

[1] I. Zutic, J. Fabian, S. D. Sarma, Spintronics: Fundamentals and applications, Rev. Mod. Phys. 76 (2004) 323. 
[2] M. Bolduc, C. Awo-Affouda, A. Stollenwerk, M. B. Huang, F. G. Ramos, G. Agnello, V. P. LaBella, Above room temperature ferromagnetism in Mn-ion implanted Si, Phys. Rev. B. 71 (2005) 033302.

[3] H. Ohno, H. Munckata, T. Penny, S. von Molnar, L. L. Chang, Magnetotransport properties of p-type (In,Mn) as dilute magnetic III-V semiconductors, Phys. Rev. Lett. 68 (1992) 2664.

[4] A. Haury, A. Wasiela, A. Arnoult, . J. Cibert, S. Tatarenko, T. Dietl, Y. M. dAubign, Observation of a ferromagnetic transition induced by two-dimensional hole gas in modulation-doped CdMnTe quantum wells, Phys. Rev. Lett. 79 (1997) 511.

[5] K. Sato, H. Katayama-Yoshida, First principles materials design for semiconductor spintronics, Semicond. Sc. Technol. 17 (2002) 367-376.

[6] P. Mahadevan, A. Zunger, Trends in ferromagnetism, hole localization, and acceptor level depth for mn substitution in GaN, GaP, GaAs, and GaSb, Applied Physics Letters 85 (2004) 2860.

[7] T. C. Schulthess, W. M. Temmerman, Z. Szotek, W. H. Butler, G. M. Stocks, Electronic structure and exchange coupling of mn impurities in iiiv semiconductors, Nature Mat. 4 (2005) 838.

[8] M. Toyoda, H. Akai, K. Sato, H. Katayama-Yoshida., Electronic structures of $(\mathrm{Zn}, \mathrm{TM}) \mathrm{O}$ (TM : V, Cr, Mn, Fe, Co, and Ni) in the selfinteraction-corrected calculations, Physica B-Condensed Matter 376 (2006) 647.

[9] H. Kizaki, M. Toyoda, K. Sato, and H. Katayama-Yoshida., FirstPrinciples Study on Electronic Structure and Spin State of Rutile (Ti, Co) $\mathrm{O}_{2}$ by Self-Interaction-Corrected Local Density Approximation: Role of Oxygen Vacancy, Appl. Phys. Express 2 (2009) 53004.

[10] M. Bolduc, C. Awo-Affouda, F. Ramos, V. P. LaBella, Annealing temperature effects on the structure of ferromagnetic mn-implanted Si, J. Vac. Sci. Technol. A. 24 (2006) 1648.

[11] M. Bolduc, C. Awo-Aouda, A. Stollenwerk, M. Huang, F. Ramos, V. LaBella, Investigation of the structural properties of ferromagnetic Mnimplanted Si, Nucl. Instr. Meth. Phys Res B 242 (2006) 367-370. 
[12] H.-T. Lin, W.-J. Huang, S.-H. Wang, H.-H. Lin, T.-S. Chin, Carriermediated ferromagnetism in p-Si(100) by sequential ion-implantation of B and Mn, J. Phys. Cond. Mat 20 (2008) 095004.

[13] C. Awo-Affouda, M. Bolduc, M. B. Huang, F. Ramos, K. A. Dunn, B. Thiel, G. Agnello, V. P. a. LaBella, Observation of crystallite formation in ferromagnetic Mn-implanted Si, J. Vac. Sci. Technol. A. 24 (2006) 1644.

[14] S. Ma, Y. Sun, B. Zhao, P. Tong, X. Zhu, W. Song, Magnetic and electronic transport properties of Mn-doped silicon, Solid. State. Commun 140 (2006) 192.

[15] K. Sato, W. Schweika, P. H. Dederichs, and H. Katayama-Yoshida., Low-temperature ferromagnetism in $(\mathrm{Ga}, \mathrm{Mn}) \mathrm{N}$ : Ab initio calculations, Phys. Rev. B 70 (2004) 201202 .

[16] T. Fukushima, K. Sato, H. Katayama-Yoshida, and P. H. Dederichs, Spinodal decomposition under layer by layer growth condition and high Curie temperature quasi-one-dimensional nano-structure in dilute magnetic semiconductors, Jap. J. Appl. Phys. Part 2-Lett. \& Exp. Lett. 45 (2006) L416.

[17] H. Katayama-Yoshida, K. Sato, T. Fukushima, M. Toyoda, H. Kizaki, V. A. Dinh, and P. H. Dederichs, Theory of ferromagnetic semiconductors, Phys. Stat. Solidi A-Appl. and Mat. Sc. 204 (2007) 15.

[18] K. Sato, H. Katayama-Yoshida, and P. H. Dederichs, High Curie temperature and nano-scale spinodal decomposition phase in dilute magnetic semiconductors, Jap. J. Appl. Phys. Part 2-Lett. \& Exp. Lett. 44 (2005) L948.

[19] H. Katayama-Yoshida, K. Sato, T. Fukushima, M. Toyoda, H. Kizaki, V.A. Dinh, P.H. Dederichs, Computational Nano-Materials Design for High-Tc Ferromagnetism in Wide-Gap Magnetic Semiconductors, J. Mag. Mag. Mat. 310 (2007) 2070.

[20] K. Sato, T. Fukushima, and H. Katayama-Yoshida, Super-Paramagnetic Blocking Phenomena and Room-Temperature Ferromagnetism in Wide Band-Gap Dilute Magnetic Semiconductor (Ga, Mn)N, Jap. J. Appl. Phys. Part 2-Lett. \& Exp. Lett. 46 (2007) L682. 
[21] F. Kwen, R. Leitsmann, F. Bechstedt, Mn and Fe doping of bulk Si: Concentration inuence on electronic and magnetic properties, Phys. Rev. B 80 (2009) 045203.

[22] Z. Z. Zhang, B. Partoens, K. Chang, F. M. Peeters, First-principles study of transition metal impurities in Si, Phys. Rev. B. 77 (2008) 155201.

[23] Q. Liu, W. Yan, H. Wei, Z. Sun, Z. Pan, A. V. Soldatov, C. Mai, C. Pei, X. Zhang, Y. Jiang, . Shiqiang Wei1, Energetic stability, electronic structure, and magnetism in Mn-doped silicon dilute magnetic semiconductors, Phys. Rev. B. 77 (2008) 245211.

[24] J. T. Arantes, A. J. R. da Silva, A. Fazzio, A. Antonelli, Theoretical investigation of a Mn-doped Si / Ge heterostructure, Phys. Rev. B. 75 (2007) 075316.

[25] H. Wu, P. Kratzer, M. Schefer, Density-functional theory study of halfmetallic heterostructures: Interstitial $\mathrm{Mn}$ in $\mathrm{Si}$, Phys. Rev. Lett. 98 (2007) 117202.

[26] H. Weng, J. Dong, First-principles investigation of transition-metaldoped group-IV semiconductors: $\mathrm{R}_{x} \mathrm{y}_{1 x} \mathrm{R}=\mathrm{Cr}, \mathrm{Mn}, \mathrm{Fe} ; \mathrm{Y}=\mathrm{Si}$ , Ge, Phys. Rev. B. 71 (2005) 035201.

[27] A. Stroppa, S. Picozzi, A. Continenza, A. J. Freeman, Electronic structure and ferromagnetism of Mn-doped group-IV semiconductors, Phys. Rev. B. 68 (2003) 155203.

[28] H. Chen, W. Zhu, E. Kaxiras, Z. Zhang, Optimization of mn doping in group-IV-based dilute magnetic semiconductors by electronic codopants, Phys. Rev. B 79 (2009) 235202.

[29] W. Zhu, Z. Zhang, E. Kaxira, Dopant-assisted concentration enhancement of substitutional Mn in Si and Ge, Phys. Rev. Lett. 100 (2008) 027205 .

[30] V. Saunders, R. Dovesi, C. Roetti, R. Orlando, C. Zicovich-Wilson, N. Harrison, K. Doll, B. Civalleri, I. Bush, P. D'Arco, M. Llunell, CRYSTAL 2006 Users's Manual, University of Torino, 2006. 
[31] J. Muscat, A. Wander, N. M. Harrison, Chem. Phys. Letts. 342 (2001) 397.

[32] X.-B. Feng, N. M. Harrison, The metal-insulator and magnetic transition of $\mathrm{NiO}$ at high pressures, Phys. Rev. B. 69 (2004) 035114.

[33] F. Beeler, O. K. Andersen, M. Scheffler., Electronic and magnetic structure of 3d-transition metal point defects in silicon calculated from first principles., Phys. Rev. B. 41 (1990) 1603.

[34] A. Kaminski, S. D. Sarma, Polaron percolation in diluted magnetic semiconductors, Phys. Rev. Lett. 88 (2002) 247202. 